\title{
Virtualized Classroom - Automated Production, Media Integration and User-Customized Presentation
}

\author{
Zhigang Zhu ${ }^{1,2 *}$, Chad McKittrick ${ }^{1}$ and Weihong $\mathrm{Li}^{2}$ \\ ${ }^{1}$ Department of Computer Science, City College, The City University of New York, New York, NY 10031 \\ ${ }^{2}$ Department of Computer Science, Graduate Center, The City University of New York, NY 10016 \\ *zhu@cs.ccny.cuny.edu
}

\begin{abstract}
Multimedia materials from classrooms and seminars are rich sources of information. Our Virtualized Classroom Project, which is a testbed for integrating novel multimedia software systems, addresses fundamental research problems occurring in different components of a next-generation, processingenhanced e-learning system. Our ultimate goal is to create a natural learning environment that could give students interactive experiences equivalent to, or even better than, the real classroom. This will be enabled by virtual instructor presence, user-selectable and fully-indexed visual aids, and flexible communications between students, the instructor and virtual instructors (computers). We have developed several basic components for the Virtualized Classroom project: automated data collection, intelligent media integration, and flexible user interfaces.
\end{abstract}

\section{Introduction}

A domain of primary importance in the future of web-based technology and digital libraries is distance and electronic education (e-learning). Multimedia materials from classrooms and seminars are rich sources of information. Today's first generation elearning systems primarily adopt a "record-andplayback" approach, which does not leverage the processing capabilities (during live capture, aftercapture processing, and during later user interaction with archived materials) that we believe will underlie the next generation of more automated, flexible, comprehensible, and individualized e-learning systems. The goal of the Virtualized Classroom project is to design novel software systems to address fundamental research problems occurring in different components of a next-generation, processing-enhanced e-learning system for college and graduate courses. It includes the following key components:

- Automatic data collection, analysis, and multimodal synchronization. In the pre-production stage, the audio/video and content information (of blackboard/ whiteboard, slide projections, digital slides, and activities of the instructor as well as students) in the classroom is collected and analyzed, using multiple sensors (cameras, microphones, screen capturer and whiteboard digitizer). Automatic camera management is performed in this stage with emphasis on three major tasks: automated camera control, media synchronization and automatic data collection.

- Intelligent post-processing: compression, crossmedia indexing and archiving. We will develop techniques to perform post-processing for the crossindexing of materials via an indexed Table of Contents of keywords and topics, and for the purposes of subsequent information search and retrieval (e.g., using cross-indexed text, audio, and image to enhance retrieval using any one of these modalities), subsequent media substitution (e.g., user replacement of PowerPoint images in video with digital representations drawn directly from the original PowerPoint slides), and subsequent user customized lecture presentation.

- Natural Interaction between students, teachers and computers. One of the main concerns of today's e-learning systems is whether deep understanding of concepts(not just the accumulation of facts) could occur in the absence of human interaction. In the Virtualized Classroom, we are aiming to create a natural environment that could improve the real classroom in stimulating broader and novel interaction, -- enabled by the concept of active media objects in the Virtualized Classroom, including: a virtual instructor presence, user-selectable and fully-indexed visual aids and materials, full communications between students, instructors and computers, all organized in the Virtualized Classroom environment that is natural and friendly.

We use the name Virtualized Classroom to indicate that the new e-learning environment is generated and enhanced from materials captured from real classrooms. The Virtualized Classroom concept could be viewed as a special application of the Virtualized Reality technique [Kanade99]. We envision that every meaningful object is active in the Virtualized Classroom. 


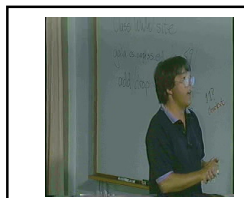

(a)

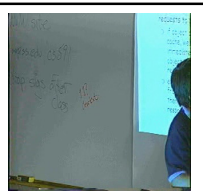

(b)

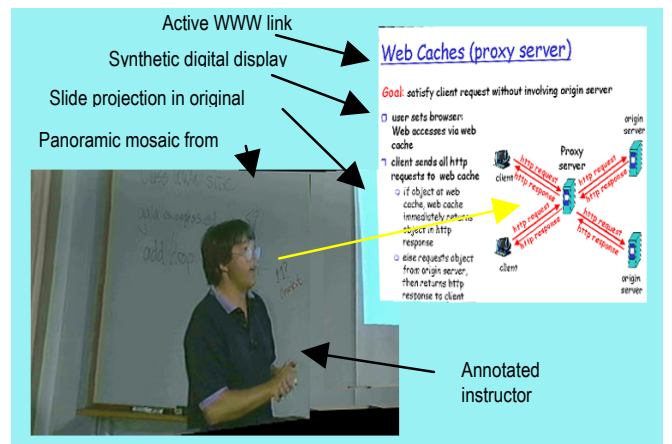

(d)

Fig. 1. Active objects and interactive user interfaces. A mosaic is generated from the panning camera tracking the instructor [Zhu99]. (a) Frame 1, (b) Frame 50 and (c) Frame 150. (d) Improved panoramic mosaic with the slide projection aligned with the high resolution digital slide. Note that active objects of the digital display enable active WWW links in the images that the user can click on.

First the image of the instructor is active not only because it is dynamic but it also is annotated and linked to his/her important information such as his/her web page, email and phone numbers. By clicking the image of the instructor, a student can find this information and may also ask questions on the spot, or make an appointment by sending him/her email, or sometimes even directly talk with him/her (if he/she is online). In addition, students may be able to engage in discussion with the instructor and other students about key points in the lecture through a discussion board in the Virtualized Classroom interface. Second the synthetic images of the digital slides and blackboard are also active with a glossary and dictionary, remedial tutorials, WWW links, and audio/video clips that may be presented in the original lecture. A Table of Contents (TOC) is active and at hand in the sense that it is floating or hiding in the $3 \mathrm{D}$ virtual space so that it can be activated whenever desired. The idea of active slides make it possible for a student to point to a term or sentence that he/she wants to learn more about it, and the system will search the notes in this lecture and across other lectures and designated courses, as well as the collateral materials on the web as specified by the instructor. The active slides are similar to HTML pages, but they are presented in the virtual space aligned with the instructor and other meaningful items in the space (Fig. 1). Finally we are going to provide an active question -answering window so that students can share their discussions with each other and the instructor.

The combination of active objects with the usercustomized interface provides effective interaction between the teacher and students, and exactly follows the principles of good teaching: for example it encourages student to faculty and student to student contact and active learning, it provides prompt feedback, and, it respects diverse ways of learning. With very cost-effective multimedia sensors, we have developed the following basic components for the Virtualized Classroom project - automated data collection, intelligent media processing and integration algorithms, and user-customized interface designs. We will describe each of the components in the following three sections. Related work will be discussed in Section 5. Then we will discuss some of the future research directions in the Virtualized Classroom. Finally we will conclude our work in Section 7.

\section{Automated Data Collection}

The commonly used classroom /lecture presentation tools are PowerPoint (PPT) slides, overhead projections, and blackboards /whiteboards. In order to digitize the classroom/lecture contents into computers, we use a low-cost Mimio digital whiteboards system to substitute the use of the blackboard. The "sensors" we are using for the classroom setting are PPT slides capturer, Mimio Virtual Ink and Sony video camera with a RemoteReality omnidirectional lens. All the sensors can be easily managed by the instructor/lecturer himself/herself.

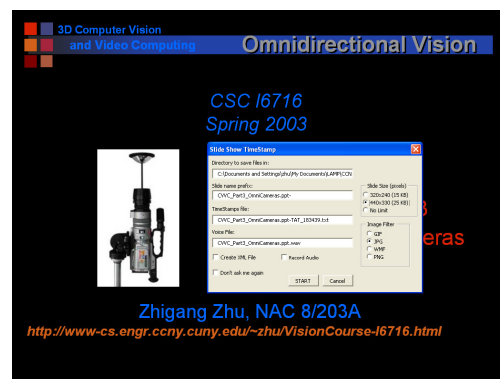

Fig. 2. Powerpoint (c slide capture. The pop-up dialog-box of the PPT slide capturer add-in is activated automatically when the instructor starts his/her PPT presentation.

\subsection{PowerPoint slide capture}

The PowerPoint slide capturer was modified from the Berkeley PPT Recording Add-In [BMRC, PINYELN]. We added the start date and time information of the presentation under recording in order to synchronize the recorded PPT slides with the 
accompanying whiteboard pages (Fig. 2). In addition to the pages of slides in one of the image formats (e.g. JPEG), a PPT log file is automatically generated with timing information and the titles of all the slides in the presentation.

\subsection{Whiteboard capture}

Everything an instructor writes on a normal whiteboard can be captured by the Mimio Virtual Ink [MVI], a hardware and software package for digitizing whiteboard handwriting and drawings (Fig. 3). The whiteboard presentation will be saved in a series of html files, one html file (with the time information of the page) for each whiteboard page (as JPEG image). An index.html file is also generated, which will be used to retrieve the whiteboard pages. The Virtual Ink system can also be used as a remote mouse and/or a remote keyboard, in that the lecturer could control his/her PPT presentation or even generate the PPT presentation just in front of the whiteboard, without touching the computer after he/she starts the system.

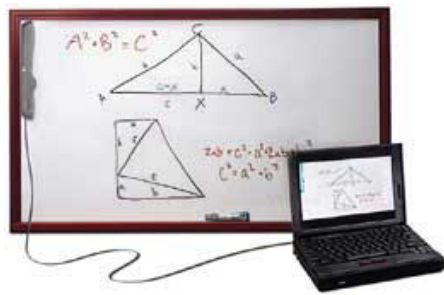

Fig. 3. Mimi@ virtual ink system is capable of recording handwriting on a normal whiteboard of $2.4 \mathrm{~m} \times 1.2 \mathrm{~m}$, with $100 \mathrm{dpi}$ resolution.

\subsection{Video / Audio Capture}

We use an omnidirectional lens developed by RemoteReality [RR] with a Sony DV camcorder to capture the entire classroom in 360 degrees so that both the instructor and students will be in the field of view (Fig. 4, Fig. 5). Of course the lecturer could just setup the DV camera to get a normal video capture. The lecturer starts recording right when he/she clicks the "OK" button in the PPT Recording Add-In pop-up panel so that the video stream will be synchronized with the slides. In our current implementation, the video stream should be saved either in an avi or in .mpg file.

If the lecturer only needs to save the audio stream, he/she just needs to set up the computer microphone, and in the PPT recording Add-In, to simply check the "Audio" check box. A .wav file will be saved, and it is synchronized with the slides.

For the best use of the above sensors, we assume that the instructor will use a computer projector to project PPT slides on a whiteboard in his/her class. The handwriting contents written on the whiteboard are on top of the slides projections, and will be captured by the Mimio Virtual Ink system. The instructor's video and audio streams will be captured by an omnidirectional camcorder. However, our Virtualized Classroom authoring tool (creator) and presentation tool (player) also work with a PPT-slides-only presentation, a whiteboard-only presentation, an audio/video-only presentation, or a combination of any of them. After the instructor sets up the sensors and starts the presentation, almost everything is automatically saved for him/her.
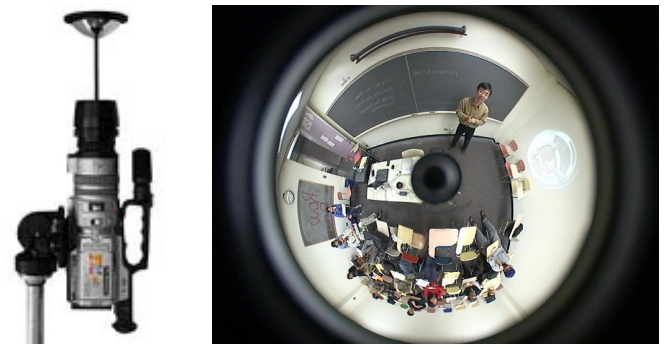

Fig. 4. The omnidirectional camera and an omnidirectional image with both the instructor and the students in view.

\section{Media Processing and Integration}

In this section, we will briefly describe several computer vision techniques in media processing and integration including panoramic viewing, media synchronization, and media integration. These techniques will be the basis for further cross-media indexing and user-controllable viewing of e-lectures.

\subsection{Panoramic video processing}

The omnidirectional camera is used to capture in real-time the 360-degree FOV classroom scene. In the video stream, both the instructor and the students can be seen. Currently we only transform an original circular video stream into a cylindrical panoramic video stream for better viewing (Fig. 5). In the future, we will study the best way to present the classroom setting using omnidirectional vision technology, for example, instructor tracking, student tracking (for question-answering and other interaction), and integration of video with slide/ whiteboard presentations.

\subsection{Media synchronization}

The synchronization of the PPT slides, whiteboard pages and audio/video streams is enabled by a simple stream synchronization algorithm which uses the timing information in the PPT log file, whiteboard log files, and the video/audio stream. There are three issues in synchronizing PPT and whiteboard page presentation. 


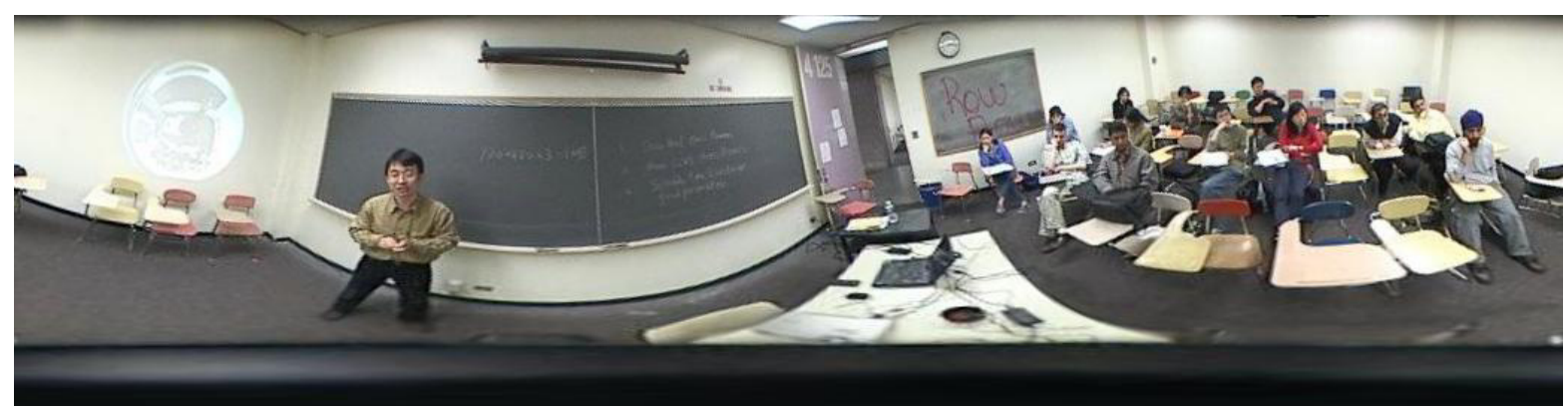

Fig. 5. Panoramic view of the classroom video. The instructor, slide projection, blackboard and students are all in the field of view.

First, each PPT slide has a start time in the presentation, while each whiteboard page has a time indicating when the page is done. Second, the number of whiteboard pages will most probably be different from the number of the PPT slides. The instructor may generate more than one white page within the presentation of a single slide, or he/she may use the same whiteboard page for several PPT slides. In other occasions, he/she may not generate any whiteboard pages for some slides. Third, the start times of the corresponding PPT slides and the whiteboard pages may be different. Our algorithm matches up the two timing sequences, taking care of all these issues.

\subsection{Registering slides and whiteboard images}

The PPT slide presentation and the whiteboard system when used together could provide a better means to present a lecture in a classroom. When the PPT projection and the whiteboard are in two separate areas, we may not need to register PPT slide images and the whiteboard images in the Virtualized Classroom presentation. However when the PPT slides are projected on the whiteboard, where the instructor is going to annotate, modify, or expend the PPT presentation, we need to geometrically register the images from the two sources to create high quality presentations. As a simple and quick approach, right after he/she starts the classroom presentation, we require the instructor to mark at least the four corners of the PPT projection in the whiteboard as a "calibration" step (Fig. 6a). The whiteboard image with the four markers is captured by the Mimio Virtual Ink and a corresponding PPT slide image is captured by PPT recorder. Since both of them are images of the same plane (the whiteboard), we use a projective transformation to register the two images with (at least) four point pairs. Typically, the sources will remain stationary (e.g., the slide projector, whiteboards) and we can use the same transformation parameters after we perform an initial registration. (Fig. 6b). For dealing with more difficult cases where portable presentation devices may be moved during a presentation, we have developed algorithms that perform dynamic registration of the two sources via a low-cost video camera that views both the slide projection and the whiteboard [Li03].

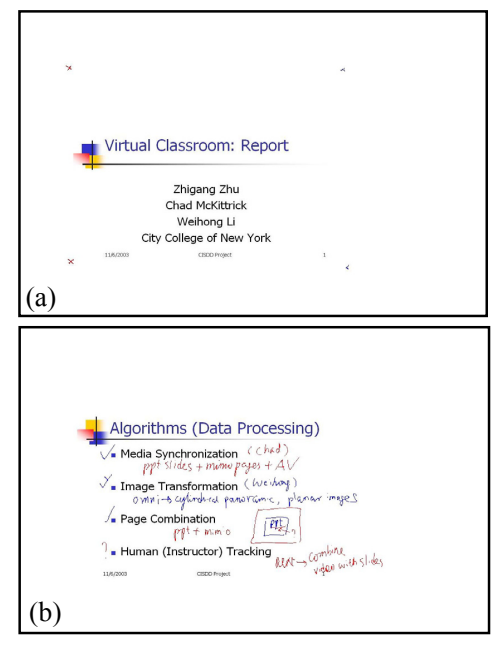

Fig. 6. Slide and whiteboard image registration. Four crosses shown in (a) are the four corners of the projection region of PPT slides. Both (a) and (b) show the size of the Mimio whiteboard active area (within the picture frames).

\subsection{Registering slides and instructor images}

The position and gestures of the instructor are a very effective way to attract the attention of students and help them to recall what they have learned in the class. In the Virtualized Classroom, actual video images of the lecturer will be merged in real time with the digital slides, so that a student will perceive the natural spatial relation between the lecturer and the visual aids. The lecturer or his/her "avatar" is in the Virtualized Classroom. For example, the lecturer may point to the items in the digital slides (Fig. 7). For this purpose, we have also done some preliminary research on automatic instructor extraction from the video streams using computer vision techniques in [Zhu00] to perform content-based video compression and slidevideo integration. As a result, the text always will be presented in sharp resolution (using the digital slides), much higher than the standard type of video. The image of the lecturer will be merged with the synthesized "slides" rather than displayed in two different windows.

Fig. 7 shows an example of automatic integration of the real and high-resolution virtual images. In (a) is a 
frame from the original video at low resolution with undesired shadows. In (b) the extracted real image of the lecturer and the synthetic image of the high resolution digital slide are merged in one view, giving the user a strong feeling that the lecturer is in the Virtualized Classroom. The slide image from video and the synthetic image from digital slide have been aligned so that the lecturer can point to the exact point that he pointed in the slide projection. In (c) the image of the lecturer is displayed as a "shadow" on the slide projection to avoid occluding the slide, while still providing a natural presence. In (d) the image of the lecturer can be replaced by a colorful contour or even simply make the item pointed to highlighted. The highlighting requires gesture recognition and hand localization.

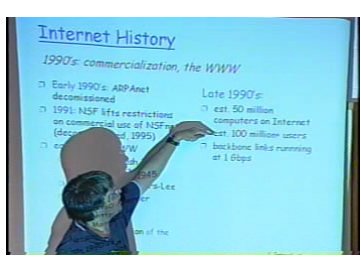

(a)

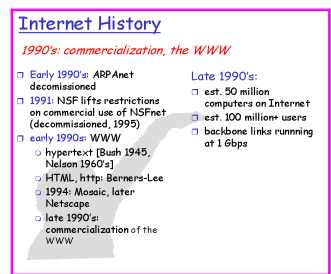

(c)

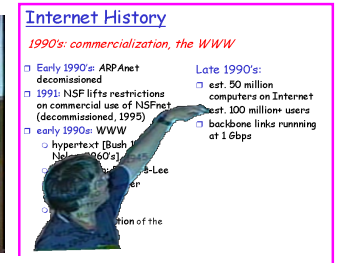

(b)

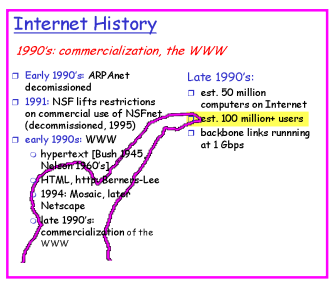

(d)
Fig. 7. Slide and video registration

\section{User-Customized Presentation}

Our current implementation of the Virtualized Classroom includes a Virtualized Classroom Presentation System (VCPS) that is designed as both an authoring tool and a presentation interface for different kinds of lectures, and has a user-selectable interface. The VCPS that is developed in Java includes two parts - the VCPS Creator and the VCPS Player.

\subsection{Virtualized Classroom Creator}

The user (an instructor or a student) who uses the system can customize the presentation by using the VCPS Creator to include different media in windows with user-selected sizes and positions. After the user opens a new Creator page, there will be a floating frame that has checkboxes on it. He/She will use these checkboxes to add or remove presentation components. Each time the user clicks on one of the checkboxes a popup window will appear directing him/her to click on a certain file so that the program can load the proper information. The media forms that have been integrated in the VCPS are the following:
- For Slides Panel, load the PPT log file (.txt)

- For Whiteboard Panel, load the whiteboard index.html file.

- For Video with Audio or Video Panel, load the mpg or avi video file (up to two video streams can be played)

- For Audio file, load the .wav file

- For Slides with Board, load the PPT log file (.txt). The VC Creator/Player assumes that the whiteboard information is in index.html file, and the combined pages (from PPT and whiteboard images) have been generated.

(a)

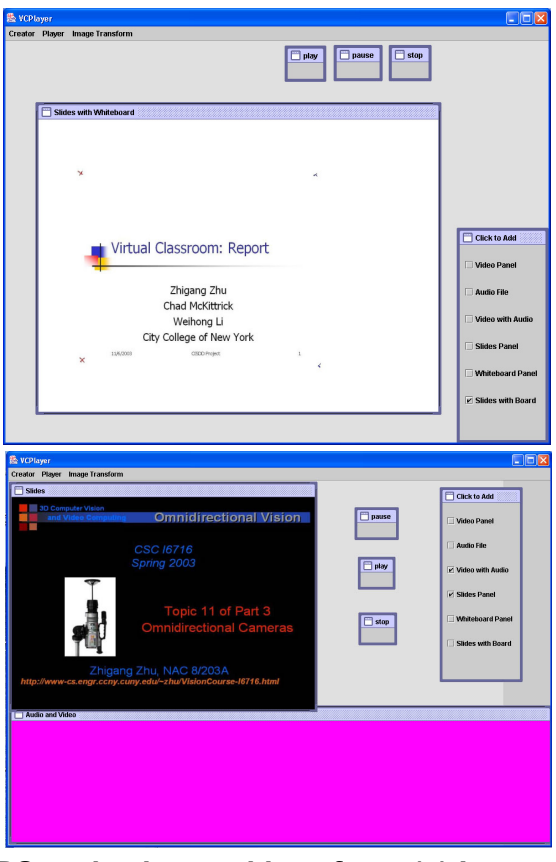

Fig. 8. VCPS authoring tool interface. (a) Integrated presentation of PPT and whiteboard pages; (b) PPT slides + panoramic video

After the user adds presentation components, the application window will contain frames encompassing the presentation. The user can resize and reposition any of the frames. Since the Creator works in a WYSIWYG fashion, the frame window will be larger than the component will be when it is played. So, even if frames overlap a bit, if the content in the frame is positioned as he/she wants, the presentation will play as he/she wants. The two snapshots in Fig. 8 show two examples with different contents, number of windows, and sizes and positions of the windows.

Once the user has included all of the components in his/her presentation, he/she need to save the presentation to a .vcp file. The operation is as simple as to go to File menu item and to click on Save, and then to chose a location in the file system, give the file any name, and click on Save in the popup window. 


\subsection{Virtualized Classroom Player}

When the user wants to play a presentation all he/she has to do is click open under the Player menu. It will take a few seconds for the presentation to load and to transform the images into the right sizes. Once it has loaded the user can click on the "Start" button to start the presentation from the beginning, or, if there are PowerPoint based slides, he/she can do a mouse right click to get a slide based Table of Contents (TOC) to guide the player to a specific topic in the presentation. All the media contents are synchronized by using the timing information (from the PPT log file, the whiteboard html file, and the video/audio timing information). Using a pop-up TOC, we can make full use of the space in the player. Fig. 9 shows snapshots of the VCPS Player interfaces. We will show live demos for both authoring and playing VCPS electures when presenting the paper.

(a)
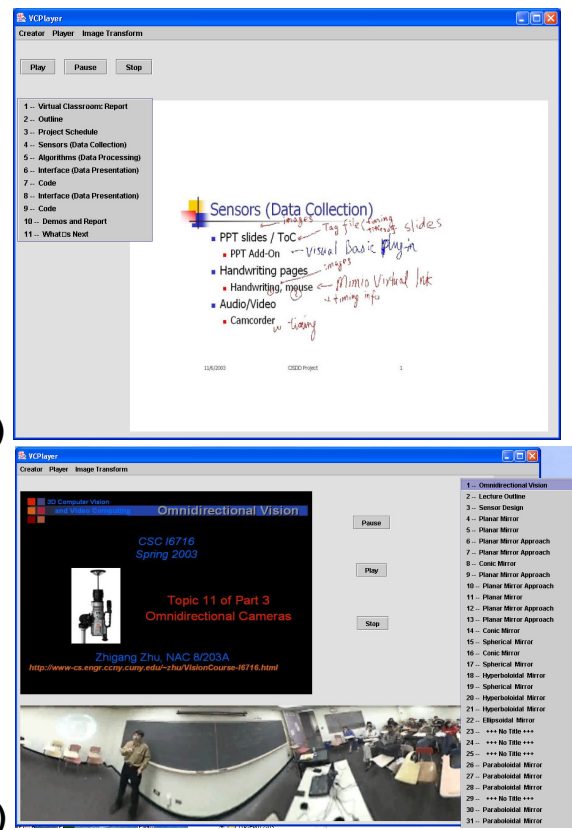

Fig. 9. VCPS Player interface. (a) Integrated presentation of PPT and whiteboard pages; (b) PPT slides + panoramic video

\section{Related Work}

Projects focused on record and playback technologies include: the MIT Physics Interactive Video Tutor project (PIVOT) [Lipson01], the Georgia Tech Classroom 2000 (eClass) [Abowd96, Abowd99], the CMU Just-In-Time system [Dannen97, JITL], and the UMass Research in Presentation Production for Learning Electronically (RIPPLES, MANIC) [Stern97], among others. Most of these produce courseware that is largely passive, typically only allowing some form of search over the text in the recorded "lectures." They generally have a limited ability to support learner navigation, which is a key to knowledge discovery and construction. Currently in existing electronic lectures, text windows, video windows, and a table of contents (TOC) are typically in a fixed format.

Active learning has been introduced in UMass RIPPLES group technologies by replacing the MANIC brute force searching with more advanced searching techniques [Kumela04] that make use of ranking and relevance, and by applying an active object interface. The new system generates the matching documents (e.g., relevant lecture segments) from within the locally available material. The content of the matching documents and the initial user query then are used to create a new query, conducted over the World Wide Web and a database of reference material using Google ${ }^{\mathrm{TM}}$. The returned links are filtered by relevance to learner interests. An "active" interface that generates queries from student interactions and the capability to query content of video and audio materials is also desirable. Several commercial speech indexing systems, including Streamsage [Sage] and Scansoft [Scansoft], have been applied to indexing on-line streamed courseware. The Informedia Digital Library project at CMU [Criste195, Wactlar99] is developing new technologies for video analysis, storage, search, and retrieval of image, video and audio content and embedding them in a distributed library system for use in education, training, sports and entertainment.

Automated production is a major issue for e-lecture systems because to improve the quality of presentations many systems require significant manual effort in analog data collection, digitization, and synchronization. In the UMass RIPPLES program, as a result of emphasizing low demand on instructors and high quality presentations, it can take five hours labor of a trained courseware developer to create a one-hour lecture. We note that some systems have introduced forms of automatic production. Cornell's lecture browser [Mukho99] includes lecturer tracking, slide change detection and segmentation, and matching slide projections with digital slides. A precursor to Cornell's lecture browser, UC Berkeley BMRC Lecture Browser [BMRC] has several additional features including audio search capabilities, bookmarks and an interactive whiteboard for online students. To enable automated production, we need a way to capture many classroom activities. MIT Media Lab's SmartCam [Pinhan95] has a robotic TV camera without a human operator, changing its attitude, zoom and position to provide specific images upon verbal request from a director. The Intelligent Classroom [Frank99, Frank00] uses cameras and microphones to determine what the speaker is trying to do and then takes the actions (e.g., jumping to a new slide) it deems appropriate. AutoAuditorium [Cruz94, Brinchi98] uses multiple cameras 
that automatically switch based on context. The automatic camera management capability (iCam) of Microsoft Research [Rui01, Liu01] automatically tracks both the speaker and the audience in a lecture room environment by using omnidirectional-visionand microphone-array-based techniques. The BlueEyes project of IBM Almaden Research Center [IBM-BE] uses non-obtrusive sensing technology, such as video cameras and microphones, to identify and observe a user's actions, and to extract key information, such as where the user is looking and what the user is saying verbally and with gestures.

\section{Discussions and Future Work}

In this section we discuss several on-going research issues that enable fast presentation creation, active navigation and natural interaction in the Virtualized Classroom.

\subsection{Multimedia active object representations}

The ultimate goal of the project is to create e-lecture presentations that include active objects in various kinds of media - PPT slides, handwriting, and video and audio so that every meaningful object in the presentation is active - terms, pictures, instructor images, and students. Collaborating with the UMass RIPPLES group, we intent to create a future Virtualized Classroom environment that supports interactive learning even though the instructor and students are neither in the same physical space nor presented in the same time. Therefore contentbased data collection and presentation is required. In the current version of our program, both the PPT slides and the whiteboard pages are saved as image files (JPEG). This does not allow cross-indexing in and among pages. In the next step, we are going to study ways to enable the implementation of the active object concept. For example we will save PPT slides into HTML files. We will save handwriting on whiteboards in their dynamic forms, and then further with handwriting recognition. We will also extract the subjects (instructor and students) and the relevant events (e.g. question \& answering) from the video sequence.

\subsection{Interactive 3D user interface design}

We will perform a feasibility study on the impact of a 3D Virtualized Classroom environment on the active learning experience. With the 3D Virtualized Classroom, we allow users to gain better understanding of the 3D spatial context of the classroom environment. We believe that this will directly improve student perception of the material or classroom presentation. We will allow the user many choices for viewing the lecture, organizing views of the blackboard, the slide, the instructor, table of contents, and supplementary materials into a spatial context.

\subsection{Usability and case study}

We will study several typical classroom examples using different instructing technologies. For example, we will apply our VCPS presentation system to different types of courses (science or art) at different levels (undergrad or graduate) and using different presentation methods (PPT presentation/ whiteboard/ blackboard/speech-only). In different cases, we will study the data collection strategy and user interface design. We will develop algorithms that can automatically collect user feedback information for assessing our Virtualized Classroom presentations.

\subsection{Real-time presentation creation}

The work to create a good PPT presentation is tedious, particularly when some fine drawings and animations are generated. Therefore we will study the feasibility of using Mimio Virtual Ink or other interactive tools to generate or to revise the PPT presentation on the fly. For doing that, we need to design better interfaces than merely the available Mimio mouse function for interactive creation of multimedia contents, and to develop algorithms for converting /combining handwriting text/drawings into PPT digital presentations.

\section{Conclusions}

In the Virtualized Classroom (VC) project, we have tested very simple yet effective techniques to automate the capturing of multimedia classroom materials, including audio/video, digital PPT slides and whiteboard writing. When we assume that the instructor uses an LCD projector to project PowerPoint $^{\circledR}$ slides on a whiteboard in his/her class, we use cost-effective sensors to collect multimedia lecture materials in the classroom, and produce very effective e-lectures. Our automated PPT Slide Capturer was modified from the Berkeley PPT Recording AddIn. Handwriting on the whiteboard (including notations "over" projected images) is captured by the Mimio Virtual Ink system. The instructor's video and audio streams are captured by an omnidirectional camcorder (in order to see the full view of the classroom for students-teacher interaction). Our Virtualized Classroom system can capture PPT-only presentations, whiteboard-only presentations, audio/video alone, or any combination of these. Once the instructor sets up the sensors and starts the presentation, almost everything is automatically saved. The synchronization of slides, whiteboard pages and audio/video streams is enabled by a stream synchronization algorithm that uses the logged timing information. A Table of Contents (TOC) is automatically generated from the 
$\log$ files. In the e-lecture presentation, the VCPS system is designed as both an authoring tool and a presentation interface for different kinds of lectures, and implements a user-selectable interface. The automated data collection/synchronization techniques and the user-customized interfaces are an important part to the overall active learning environment in the Virtualized Classroom. Our goal is to design a next generation e-learning system that is cost-effective in creating content, and low impact on instructors, while improving learning effectiveness (e.g., retention and student satisfaction) and presentation creation efficiency.

\section{Acknowledgements}

This work is partially supported by the CUNY Institute for Software Development and Design (CISDD) and the CUNY Graduate Research Technology Initiative (GRTI). We would like to thank Prof. Rick Adrion, Prof. Jim Kurose and Prof. Edward Riseman at the University of Massachusetts at Amherst for the fruitful discussions and collaboration in this project. Our thanks are also given to Dr. Parviz Kermani for sharing with us his experience and tools used in his e-learning lecture navigator (ELN) at the Polytechnic University of New York.

\section{References}

[Abowd96] G. Abowd, et. al., Teaching and Learning as Multimedia Authoring: The Classroom 2000 Project," Proc. ACM Multimedia '96, Boston MA, Nov 1996

[Abowd99] Gregory D. Abowd, Classroom 2000: An Experiment with the Instrumentation of a Living Educational Environment, IBM Systems Journal, Special issue on Pervasive Computing, 38(4), 508-530, Oct. 1999

[Bianchi98] M. Bianchi, "AutoAuditorium: a Fully Automatic Multi-Camera System to Televise Auditorium Presentations, Proceedings of the Joint DARPA/NIST Smart Spaces Workshop, Gaithersburg, MD, July 1998.

[BMRC] BMRC Lecture Browser download at http://bmrc.berkeley.edu/frame/projects/lb/

[Christe195] M. Christel, T. Kanade, M. Mauldin, R. Reddy, M. Sirbu, S. Stevens, and H. Wactlar, Informedia Digital Video Library. Communications of the ACM, 38(4), 1995

[Cruz94] G. Cruz and R. Hill, "Capturing and Playing Multimedia Events with STREAMS," Proc. ACM Multimedia, San Francisco, CA, Oct. 1994

[Dannen97] R. B. Dannenberg, “Are Just-In-Time Lectures Effective At Teaching?" June 1997 http://www.jitl.cs.cmu.edu/effectiv.htm

[Frank00] D. Franklin, S. Bradshaw, and K. Hammond. Jabberwocky: You don't have to be a rocket scientist to change slides for a hydrogen combustion lecture. Proc. Int. Conf. on Intelligent User Interfaces, 2000.

[Frank99] D. Franklin, J. Flachsbart, K. Hammond. The Intelligent Classroom. IEEE Intelligent Systems, 14(5),
IEEE, Sept.-Oct. 1999. p.2-5.

[IBM-BE] IBM BlueEyes web page: http://www.almaden.ibm.com/cs/blueeyes/

[JITL] Just-In-Time Lectures, http://www.jitl.cs.cmu.edu/

[Kanade99] T. Kanade, P. Rander, S. Vedula, and H. Saito, Virtualized Reality: Digitizing a 3D Time-Varying Event As Is and in Real Time, Mixed Reality, Merging Real and Virtual Worlds, Y. Ohta, H. Tamura ed., SpringerVerlag, 1999: 41-57

[Kumela04] D. Kumela, K. Watts and W. R. Adrion, Supporting Constructivist Learning in a Multimedia Presentation System, submitted to the Frontiers in Education Conference, Savannah, GA October 2004

[Li03] W. Li, H. Tang and Z. Zhu, Automated registration of high-resolution images from slide presentations and whiteboard handwritings via a low-cost digital video camera. The $2^{\text {nd }}$ IEEE Workshop on Image and Video Registration (with CVPR'04), July 2, 2004

[Liu01] Q. Liu, Y. Rui, A. Gupta and J.J. Cadiz, Automating Camera Management in Lecture Room Environments, Proc. ACM CHI, Seattle, WA, 2001

[MVI]. Mimio Virtual Ink, http://www.mimio.com/index.shtml

[Mukho99] S. Mukhopadhyay and B. Smith, Passive Capture and Structuring of Lectures, Proc. ACM Multimedia, Orlando. FL, October 1999

[Nayar98] V. Peri and S. Nayar, Generation of Perspective and Panoramic Video from Omnidirectional Video, Proc. DARPA Image Understanding Workshop, 1997.

[Pinhan95] C. Pinhanez and A. Bobick, Intelligent studios: using computer vision to control TV cameras, Proc. IJCA'95 Workshop on Entertainment and AI/Alife, Montreal, Canada, August, 1995.

[PINY-ELN]. PINY ELN: e-Learning Lecture Navigator, http://westlabp4-7.poly.edu/webpage/index.htm

[Rui01] Y. Rui, L. He, A. Gupta and Q. Liú, Building an Intelligent Camera Management System, Proc. Multimedia, Ottawa, Canada, Sept-Oct. 2001

[RR]. RemoteReality, http://www.remotereality.com/

[Sage] Streamsage, http://www.streamsage.com/

[Scansoft] Scansoft, http://www.scansoft.com/

[Stern97] M. Stern, J. Steinberg, H.I. Lee, J. Padhye, J. Kurose, MANIC: Multimedia Asynchronous Networked Individualized Courseware, Proc. Educational Multimedia and Hypermedia, 1997.

[Wactlar99] H. Wactlar, et al, Lessons learned from the creation and deployment of a terbyte digital video library, IEEE Computer 32(2): 66-73, 1999.

[Zhu00] Z. Zhu, K. D. Rajasekar, E. Riseman, A. Hanson. Panoramic Virtual Stereo Vision of Cooperative Mobile Robots for localizing 3D Moving Objects. Proc. IEEE Workshop on Omnidirectional Vision, Hilton Head Island, June, 2000:29-36

[Zhu99] Z. Zhu, G. Xu, E. M. Riseman, A. R. Hanson, Fast Generation of Dynamic and Multi-Resolution 360 Panorama from Video Sequences, Proc. IEEE ICMCS'99, Florence, June 7-11, 1999, vol 1, 400-406. 